\title{
Adaptive Antenna Array with weight and antenna space control
}

\author{
Kenta Umebayashi*§, Yoshimasa Kimoto*, Antti Tölli ${ }^{\dagger}$, \\ ${ }^{*}$ Department of Electrical and Electronic Engineering, Tokyo University of Agriculture and Technology, Japan \\ ${ }^{\dagger}$ Centre for Wireless Communications, University of Oulu, Finland \\ Emails: ume_k@cc.tuat.ac.jp $p^{\S}$
}

\begin{abstract}
A typical adaptive antenna array based on weight control (AAA-W) with $M$ antennas can suppress $M-1$ interference signals. In this paper, we propose AAA based on not only weight but also antenna spacing control (AAA-WS) and investigate the basic performance of AAA-WS under the line-ofsight. At first, we show that AAA-WS with two antennas $(M=2)$ can sufficiently suppress more than two interference signals while the desired signal is enhanced. The inherent interference suppression capability of AAA-WS can be determined by the maximum antenna spacing and this fact is exhibited by analysis and Monte Carlo simulations. In addition, we will show that AAA-WS with two antennas can outperform AAA-W with more than two antennas. It is notable that the additional gain in AAAWS compared to AAA-W can be around $18 \mathrm{~dB}$.

Index Terms-Adaptive antenna array, Antenna space control, Beamforming, Interference suppression, Line of sight
\end{abstract}

\section{INTRODUCTION}

Future mobile communication systems, such as $5 \mathrm{G}$, will require ever-higher data rate and reliability [1], [2]. In order to accomplish the requirements, significant attention has been paid in multiple antenna based signal processing techniques, such as adaptive antenna array (AAA) and multiple-input and multiple-output (MIMO) [3], [4]. In adaptive antenna array systems, the achievable data rate and reliability depends on the number of antennas. Specifically, a typical adaptive antenna array based on weight control (AAA-W) with $M$ antennas can inherently cancel $M-1$ interference signals [5]. However, a large number of antennas leads to high implementation cost and power consumption since it requires $M$ radio frequency (RF) circuits and analog to digital converters (ADCs)/digital to analog converters (DACs) [6].

In AAA, antenna geometries have been investigated to improve the performance of the AAA, such as interference suppression and desired signal enhancement capabilities. In [7], [8], it has been confirmed that a certain non-uniform linear array (NULA) antenna can give performance gain over uniform linear array (ULA) antenna for a given cell deployment and terminal locations, especially with narrow angular spread scenario. Minimum redundancy array based adaptive beamforming was investigated in [9]-[12]. MRA can provide a benefit to the beamforming when an interference signal is located in close angular proximity to the direction of the desired signal [9]. The investigations of antenna placements indicates a presence of suitable antenna placement for given directions of desired and interference signals. In [13], antenna placement in line-of-sight scenario is also investigated with single desired and interference signal components. In [14], the authors have extended their work to a general case in which the number of desired signals and interference signals can be more than one. The possible antenna placements are selected from uniform linear array (ULA) antenna [13], [14].

In this paper, we propose a novel type of adaptive antenna array, i.e., adaptive antenna array based on weight and spacing control (AAA-WS), in which not only the weight but also antenna spacing are controllable. Specifically, we study AAAWS with two antennas under LoS [13], [14]. The number of null points in the beam pattern provided by the AAA-WS is determined by the antenna spacing and the location of the null points can be controlled by the weights. Therefore, the AAAWS with two antennas can not only suppress more than two interference signals sufficiently, but also enhance one desired signal. This fact inherently indicates that a gain provided by the degree of freedom in the antenna spacing is more than a gain provided by the degree of freedom in single antenna. The other contributions of this paper are summarized as follows.

- We exhibit that inherent performance of the AAA-WS is determined by the maximum antenna spacing. Specifically, larger maximum antenna spacing has more ability to suppress the interference signals. We will proof this fact based on analysis and Monte Carlo simulations.

- Numerical evaluations will present advantages of AAAWS. AAA-WS can suppress more than $M$ interference signals. Furthermore, AAA-WS with $M=2$ significantly outperform AAA-W with more than two antennas. Specifically achievable gain due the antenna spacing can be about $18 \mathrm{~dB}$ in signal-to-interference-plus-noise ratio (SINR).

\section{ADAPTIVE ANTENNA ARRAY BASED ON WEIGHT AND SPACING CONTROL (AAA-WS)}

\section{A. System model}

The assumed system model of receiver with AAA-WS is shown in Fig. 1. There are one desired signal source, $N$ interference signal sources, and LoS environment. The receiver is equipped with two antennas in AAA-WS in which the antenna spacing $d$ is controllable as $0<d \leq L$ where $L$ is the maximum antenna spacing. 


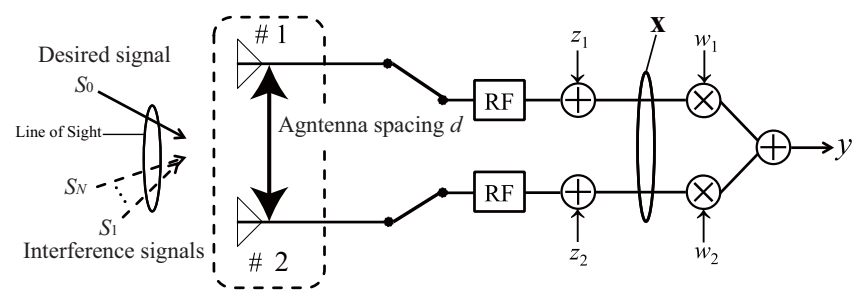

Fig. 1. System model of AAA-WS based receiver

The received signal vector $\mathbf{x}$ is defined by:

$$
\mathbf{x}=\sum_{n=0}^{N} \sqrt{p_{n}} s_{n} \mathbf{a}\left(d, \theta_{n}\right)+\mathbf{z}
$$

where $n$ denotes the index number for signal while $n=0$ represents the desired signal, $p_{n}$ denotes the received signal power, $\theta_{n}$ is the direction of arrival, $s_{n}$ denotes the signal component with unit power, $\mathbf{z}=\left[z_{1}, z_{2}\right]^{T}$ is an additive white Gaussian noise vector in which each component follows an independent circularly symmetric complex Gaussian random variable, i.e., $z_{i} \sim C N\left(0, P_{z}\right)$, and $\mathbf{a}\left(d, \theta_{n}\right)$ is steering vector for $n$th source as:

$$
\mathbf{a}\left(d, \theta_{n}\right)=\left[1, \exp \left(-j 2 \pi \frac{d}{\lambda} \sin \theta_{n}\right)\right]^{T},
$$

where $[\cdot]^{T}$ is a transpose function. The output of AAA $y$ is as

$$
y=\mathbf{w}^{H} \mathbf{x}
$$

where $\mathbf{w}=\left[w_{1}, w_{2}\right]^{T}$ is a weight vector and $H$ denotes a conjugate transposition function. Output signal power for the $n$th signal source is given by:

$$
p_{\text {out }, n}(d)=\frac{1}{2} p_{n}\left|\mathbf{w}^{H} \mathbf{a}\left(d, \theta_{n}\right)\right|^{2} .
$$

Achievable SINR as a function of $d$ is as follows:

$$
\gamma(d)=\frac{p_{\text {out }, 0}(d)}{\sum_{n=1}^{N} p_{\text {out }, n}(d)+\frac{1}{2} P_{z} \mathbf{w}^{H} \mathbf{w}},
$$

while the input signal to noise power ratio (SNR) and signal to interference signal power ratio (SIR) are defined by

$$
\gamma_{\mathrm{SNR}_{\mathrm{in}}}=\frac{p_{0}}{P_{z}}
$$

and

$$
\gamma_{\mathrm{SIR}_{\mathrm{in}}}=\frac{p_{0}}{\sum_{n=1}^{N} p_{n}}
$$

respectively.

We investigate two criteria, i.e. minimum mean square error (MMSE) and maximum ratio combiner (MRC), for setting the weight and antenna spacing. A weight vector based on the MMSE criterion is given by

$$
\mathbf{w}_{\text {MMSE }}=p_{0} \mathbf{R}^{-1} \mathbf{a}\left(d, \theta_{0}\right),
$$

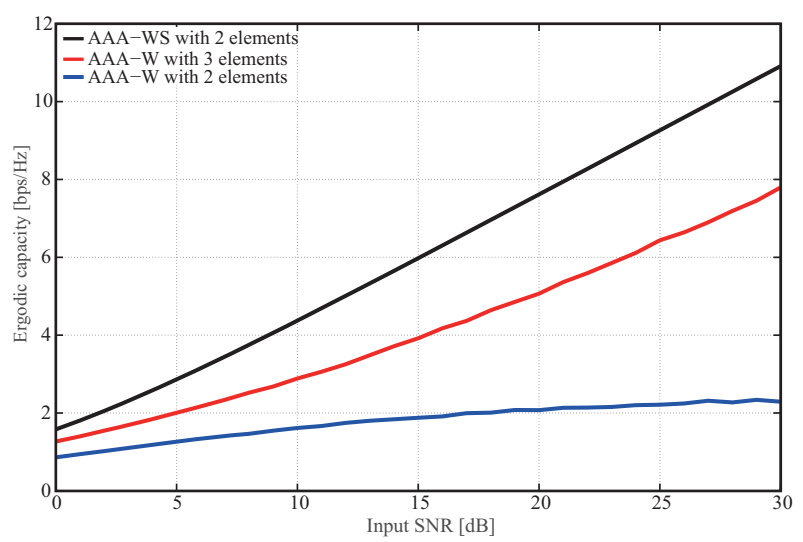

Fig. 2. Ergodic capacity of AAA-WS $(M=2)$, AAA-W ( $M=2$ and $M=3$ ), as a function of input SNR: input $\mathrm{SIR}=0 \mathrm{~dB}, N=2, L=200 \lambda$

where

$$
\mathbf{R}=\sum_{n=1}^{N} p_{n} \mathbf{a}\left(d, \theta_{n}\right) \mathbf{a}\left(d, \theta_{n}\right)^{H}+P_{z} \mathbf{I}
$$

and $\mathbf{I}$ is $2 \times 2$ identity matrix. In the MMSE criterion, the achievable SINR is given by

$$
\begin{aligned}
& \gamma_{\mathrm{MMSE}}(d)=p_{0} \mathbf{a}^{H}\left(d, \theta_{0}\right) \mathbf{R}_{n}^{-1} \mathbf{a}(d, \theta) \\
& =\frac{2 p_{0}\left[\sum_{n=1}^{N} p_{n}\left(1-\cos \left\{2 \pi f_{n 0} \frac{d}{\lambda}\right\}\right)+P_{z}\right]}{2 P_{z} \sum_{n=1}^{N} p_{n}+2 \sum_{k=1}^{N-1} \sum_{l=k+1}^{N} p_{k} p_{l}\left\{1-\cos 2 \pi f_{k l} \frac{d}{\lambda}\right\}+P_{z}^{2}} .
\end{aligned}
$$

where $f_{k l}=\sin \theta_{k}-\sin \theta_{l}$. A weight vector based on the MRC criterion is given by

$$
\mathbf{w}_{\mathrm{MRC}}=\mathbf{a}\left(d, \theta_{0}\right) .
$$

Achievable SINR with MRC weight is as follows

$$
\gamma_{\mathrm{MRC}}(d)=\frac{2 p_{0}}{\sum_{n=1}^{N} p_{n}\left(1+\cos 2 \pi f_{n 0} \frac{d}{\lambda}\right)+P_{z}}
$$

Optimum antenna spacings for MMSE and MRC criteria are defined by

$$
d_{\mathrm{MMSE}}^{\mathrm{OPT}}=\arg \max _{0<d \leq L} \gamma_{\mathrm{MMSE}}(d),
$$

and

$$
d_{\mathrm{MRC}}^{\mathrm{OPT}}=\arg \max _{0<d \leq L} \gamma_{\mathrm{MRC}}(d),
$$

respectively.

\section{B. Comparison between $A A A-W S$ and $A A A-W$}

In this section, at first we will show the advantage of AAA-WS compared to AAA-W. Fig. 2 shows the ergodic capacity for AAA-WS and AAA-W as a function of the input $\operatorname{SNR}\left(\gamma_{\mathrm{SNR}_{\text {in }}}\right)$. In this evaluation, it is assumed that 


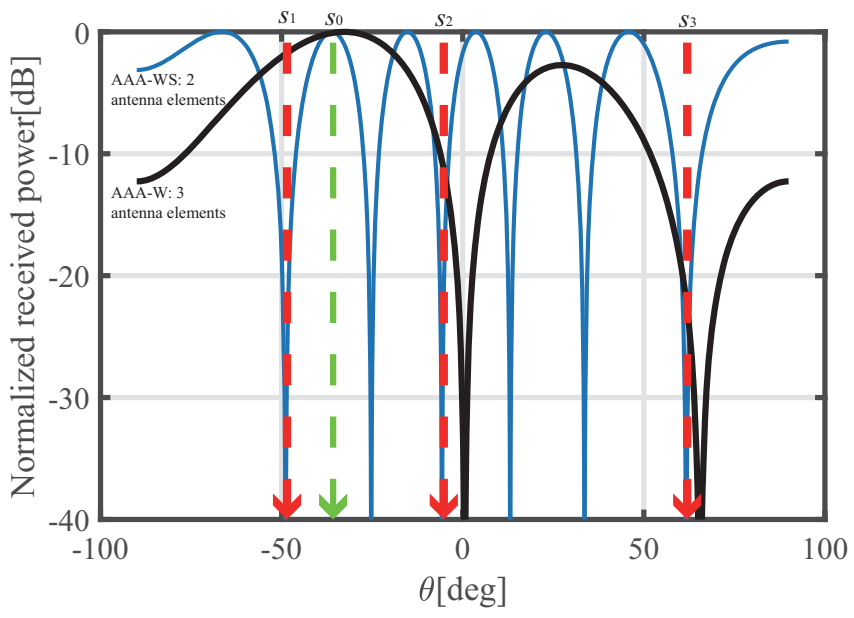

Fig. 3. Beam Pattern, $N=3$, SNR 20[dB], INR 20[dB], direction of desired signal $\left(s_{0}\right)$ is $\theta_{0}=-38.2[\mathrm{deg}]$, and directions of interference signals $\left(s_{1}, s_{2}\right.$, and $\left.s_{3}\right)$ are $\theta_{1}=-49.5[\mathrm{deg}], \theta_{2}=-6.1[\mathrm{deg}]$, and $\theta_{3}=57.3[\mathrm{deg}]$

$\gamma_{\mathrm{SIR}_{\text {in }}}=0 \mathrm{~dB}, N=2$. The direction of arrival $\theta_{n}$ follows uniform distribution in the region where $-\pi<\theta_{n}<\pi$. For the weight setting in AAA-WS and AAA-W, the MRC criterion is employed ${ }^{1}$. In AAA-WS, $d_{\mathrm{MRC}}^{\mathrm{OPT}}$ is also used. The ergodic capacity for AAA-WS is approximated by averaged $\log \left(1+\gamma_{\mathrm{MRC}}\left(d_{\mathrm{MRC}}^{\mathrm{OPT}}\right)\right)$ in terms of the direction of arrivals. In AAA-W, the antenna spacing is set to a half wave length $(\lambda / 2)$. The ergodic capacity for AAA-W is approximated by averaged $\log \left(1+\gamma_{\mathrm{MRC}}(\lambda / 2)\right)$.

In the case of AAA-W with two antennas, it does not have sufficient antenna degree of freedom to cancel the two interference signals. Therefore, in Fig. 2, the ergodic capacity of AAA-W with two antennas is saturated at about $2 \mathrm{bps} / \mathrm{Hz}$ in the high $\gamma_{\mathrm{SNR}_{\text {in }}}$ region. On the other hand, AAA-W with three antennas introduces suitable antenna degree of freedom while the ergodic capacity is increased by increasing $\gamma_{\mathrm{SNR}_{\mathrm{in}}}$. In the case of three antennas, there is also additional power gain. AAA-WS with two antennas can achieve the best ergodic capacity performance and the achievable gain is significant compared to the AAA-Ws. This fact indicates that the degree of freedom in the antenna spacing can provide more gain than the gain provided by one antenna.

The reason that AAA-WS with two antennas can suppress more than $M-1$ interference signals is expressed as follows. Fig. 3 shows beam patterns obtained by AAA-W $(M=3)$ and AAA-WS $(M=2)$. In this example, it is assumed that $N=4$ and one desired signal $s_{0}$ and three interference signals $s_{1}$, $s_{2}$, and $s_{3}$. The AAA-W with three antennas does not have enough number of antennas for suppressing the interference signals and it is harmed by the interference signals. In this example, the interference signal $\left(s_{1}\right)$ from $\theta_{1}=-49.5$ [deg] is not suppressed sufficiently in the AAA-W with three antennas.

\footnotetext{
${ }^{1}$ The reason of using the MRC is that an intuitive explanation to understand the advantage of AAA-WS is straightforward compared to MMSE. An evaluation with MMSE will be shown in Sect.V-C.
}

On the other hand, the AAA-WS can suppress all of the interference signals with $20 \mathrm{~dB}$ attenuation. The beam pattern $P(\theta)$ in the AAA-WS is given by:

$$
\begin{aligned}
P(\theta) & =\frac{\left|\mathbf{w}^{H} \mathbf{a}(d, \theta)\right|^{2}}{\mathbf{w}^{H} \mathbf{w}} \\
& =1+2 \frac{A_{0} A_{1}}{A_{0}^{2}+A_{1}^{2}} \cos \left\{2 \pi \frac{d}{\lambda} \sin \theta-\left(\phi_{0}-\phi_{1}\right)\right\} \\
& \text { where } \mathbf{w}=\left[\begin{array}{l}
A_{0} e^{j \phi_{0}} \\
A_{1} e^{j \phi_{1}}
\end{array}\right] .
\end{aligned}
$$

Specifically, when $A_{0}=A_{1}$ and $\phi_{0}=0$, the beam pattern is given by:

$$
P(\theta)=1+\cos \left\{2 \pi \frac{d}{\lambda} \sin \theta+\phi_{1}\right\} .
$$

The beam pattern $P(\theta)$ indicates the inherent aspect of the AAA-WS. The antenna spacing $d$ determines the number of nulls in the beam pattern and it is approximately $2 d / \lambda$. AAAWS with a given $d$ determines a rough shape of the beam pattern. The weight can control $\phi_{1}$ in $P(\theta)$ and it shifts the beam pattern with the given $d$ in a direction of horizontal axis in Fig. 3. Finally, the AAA-WS can suppress the interference signals and enhance the desired signal efficiently as shown in Fig. 3.

\section{INTERFERENCE SUPPRESSION ABILITY OF AAA-WS}

In this section, we show a fact that an inherent interference suppression ability of the AAA-WS is determined by the maximum antenna space $L$. We assume the MRC criterion (11) for weight setting as:

$$
\mathbf{w}_{\mathrm{MRC}}=\left[1, \exp \left(-j 2 \pi \frac{d}{\lambda} \sin \theta_{0}\right)\right]^{T} .
$$

In this case, the interference signal power of $n$th interference signal as a function of $d$ is as

$$
p_{\text {out }, n}(d)=p_{n}\left[1+\cos \left\{2 \pi \frac{d}{\lambda}\left(\sin \theta_{n}-\sin \theta_{0}\right)\right\}\right] \text {. }
$$

The equation (19) indicates that $p_{\mathrm{out}, n}(d)$ is a periodic function in terms of $d$ and it can achieve $p_{\text {out }, n}(d)=0$ with multiple antenna spacings. Let $\mathbf{d}_{n}$ denote a set of the antenna spacings, which can achieve $p_{\text {out }, n}(d)=0$ as:

$$
\mathbf{d}_{n}=\left\{d_{m}^{(n)} \mid d_{m}^{(n)}=(2 m-1) \alpha_{n}, m \in \mathbb{N}\right\},
$$

where $\alpha_{n}=\lambda /\left(2\left|\sin \theta_{n}-\sin \theta_{0}\right|\right)$. Alternatively, (19) can be written as:

$$
\begin{aligned}
p_{\text {out }, n}(d) & =p_{n}\left[1-\cos \left\{2 \pi \frac{d_{m}^{(n)}-d}{\lambda}\left(\sin \theta_{n}-\sin \theta_{0}\right)\right\}\right] \\
& =p_{n}\left[1-\cos \left\{\delta_{m}(d)\right\}\right] .
\end{aligned}
$$

In $(21), \delta_{m}(d)$ is defined by

$$
\delta_{m}(d)=\frac{2 \pi}{\lambda}\left|\left(d_{m^{*}}^{(n)}-d\right)\left(\sin \theta_{n}-\sin \theta_{0}\right)\right|
$$


where

$$
m^{*}=\underset{m}{\arg \min }\left|d_{m}^{(n)}-d\right|
$$

and the region of $\delta_{n}(d)$ is $0 \leq \delta_{n}(d)<\pi$. In addition, $p_{\text {out }, n}(d)$ is a monotonically increasing function in terms of $\delta_{n}(d)$ and smaller $\delta_{n}(d)$ leads to smaller interference signal power for the $n$th interference signal. The total interference signal power is given by:

$$
p_{\sum^{N}}(d)=\sum_{n=1}^{N} p_{\text {out }, n}(d) .
$$

The interference suppression ability for a given $L$ is indicated by the minimum total interference signal power as:

$$
P_{\min }(L)=\min _{0<d \leq L} p_{\sum^{N}}(d) .
$$

Let $\mathbf{d}_{\text {irr }}$ denote a set of irrational number of $d$ in the region of $0<d \leq L$. Let $\boldsymbol{\Delta}(L)$ denote a set of a vector $\left[\delta_{1}(d), \cdots \delta_{N}(d)\right]^{T}$ for an irrational number of $d$. We also define $\boldsymbol{\Delta}(L)$ as a set of a vector $\left[\delta_{1}(d), \cdots \delta_{N}(d)\right]^{T}$ with $d$ in $\mathbf{d}_{\text {irr. }}$. In fact, $\Delta(L)$ is a bijection function of $d$ in the set $\mathbf{d}_{\text {irr }}$ according to Weyl's equidistribution theorem [15]. Therefore, if $L_{1}<L_{2}, \boldsymbol{\Delta}\left(L_{1}\right) \subset \boldsymbol{\Delta}\left(L_{2}\right)$. This indicates that larger $L$ can provide more possible $\left[\delta_{1}(d), \cdots \delta_{N}(d)\right]^{T}$ in $\boldsymbol{\Delta}$. Therefore, larger $L$ inherently has more interference suppression ability in the AAA-WS.

\section{ANALYSIS OF INTERFERENCE SUPPRESSION ABILITY}

In this section, we analyze the interference suppression ability of AAA-WS in terms of the maximum antenna spacing $L$. Specifically, probability density function (PDF) of $P_{\min }(L)$ will be shown, while it assume that the possible number of antenna spacings is finite and the direction arrival $\theta_{n}$ follows independent and identically distributed (i.i.d.) uniform distribution. Assuming $\delta_{n}(d)$ also follows i.i.d. uniform distribution in $(0, \pi]$, the $p_{\text {out }, n}$ in $(21)$ is given by

$$
f\left(p_{\text {out }, n}\right)=\frac{1}{\pi \sqrt{p_{n}^{2}-\left(p_{\text {out }, n}-p_{n}\right)^{2}}},
$$

where $p_{\text {out }, n}$ is independent from $d$ due to (21). The derivation details of $f\left(p_{\text {out }, n}\right)$ is shown in Appendix A. Now we assume that $p_{\text {out }, n_{1}}$ and $p_{\text {out, } n_{2}}$ are independent if $n_{1} \neq n_{2}$. To simplify the notation, now $\alpha$ denotes $p_{\sum^{N}}$ in the rest of this paper. PDF of $\alpha, f(\alpha)$, can be obtained by the convolution of $f\left(p_{\text {out }, n}\right)[16]$.

In the case of $N=2$ and $p_{1}=p_{2}$, a closed form of $f(\alpha)$ is available as:

$$
\begin{aligned}
& f(\alpha)=\frac{1}{2} \frac{\Gamma(1)}{\Gamma^{2}(0.5)}\left\{1-\alpha /\left(2 p_{1}\right)\right\}^{0.5} \\
& \cdot F_{1}\left(0.5,0.5,0.5 ; 1 ; \frac{\alpha}{\alpha-2 p_{1}}, \frac{\alpha}{2 p_{1}}\right)
\end{aligned}
$$

when $0 \leq \alpha \leq p_{1}$ and

$$
\begin{aligned}
& f(\alpha)=\frac{1}{2} \frac{\Gamma(1)}{\Gamma^{2}(0.5)}\left\{\alpha /\left(2 p_{1}\right)-1\right\}^{0.5} \\
& \cdot F_{1}\left(0.5,-0.5,-0.5 ; 1 ; 2-\frac{\alpha}{\alpha-2 p_{1}}, \frac{4 p_{1}-\alpha}{2 p_{1}-\alpha}\right),
\end{aligned}
$$

when $p_{1}<\alpha \leq 2 p_{1}$, where $F_{1}$ is Appell's first hypergeometric function as:

$$
\begin{aligned}
& F_{1}\left(a, b_{1}, b_{2} ; c ; x_{1}, x_{2}\right)= \\
& \sum_{n=0}^{\infty} \sum_{m=0}^{\infty}[A(a, m+n) / A(c, m+n)] A\left(b_{1}, m\right) A\left(b_{2}, n\right) \\
& \cdot A\left(x_{1}^{m} / m !\right) A\left(x_{2}^{n} / n !\right)
\end{aligned}
$$

where $A(a, m)=\Gamma(a+m) / \Gamma(a), m \geq 0$, and $\Gamma()$ is the Gamma function. Now, we define a set of antenna spacings

$$
\mathbf{d}=\left\{d_{0}, d_{1}, \cdots, d_{k}, \cdots, d_{K}\right\},
$$

where $d_{K}=L$ and $0<d_{k} \leq L$. In this case, $P_{\min }(L)$ is given by

$$
P_{\min }(L)=\min _{k \in\{1,2, \cdots K\}} p_{\sum^{N}}\left(d_{k}\right) .
$$

According to the order statistic [17], PDF of the minimum $P_{\text {min }}(L)$ from the $K$ antenna spacings is given by:

$$
f\left(\alpha=P_{\min }(L) ; K\right)=K(1-F(\alpha))^{K-1} f(\alpha),
$$

where $F(\alpha)$ denotes a cumulative distribution function (CDF) of $f(\alpha)$. The assumption of finite number of $d$ can be expressed by $\mathbf{d}$. One possible approach to realize the finite number of $d$ in the AAA-WS is antenna selection from $K$ possible antenna spacings [13], [14]. In this analysis, $K$ possible $p_{\sum^{N}}(d)$ are assumed to be independent each other. Let $\Delta d$ denotes $\Delta d=\left|d_{k}-d_{k-1}\right|$ and $\Delta d$ is set to achieve low correlation in terms of $p_{\sum^{N}}(d)$, such as $\Delta d=0.4 \lambda$ and $L=\Delta d K$.

In the case of $N>2$ or $p_{1} \neq p_{2}$, a numerical calculation can provide the $\mathrm{PDF}$ of the minimum $P_{\min }(L)$ and $f(\alpha)$.

\section{NUMERICAL EVALUATION}

At first, we will show that the interference suppression ability of AAA-WS can be determined by $L$ by numerical evaluation. Next, a validity of the analysis discussed in Sect. IV will be confirmed. Specifically, outage probability obtained from PDF of $P_{\min }(L)$ is evaluated. In the above two evaluations, we employ the MRC criterion. Finally, performance comparison between AAA-W and AAA-WS with MMSE criterion in terms of mean of output SINR will be shown. This evaluation can show achievable SINR output gain.

\section{A. Interference suppression ability}

An event $A(\eta)$ with a coefficient $\eta$ is defined by

$$
A(\eta)= \begin{cases}1 & P_{\min }(L)<\eta \sum_{n=1}^{N} p_{n} \\ 0 & P_{\min }(L) \geq \eta \sum_{n=1}^{N} p_{n}\end{cases}
$$




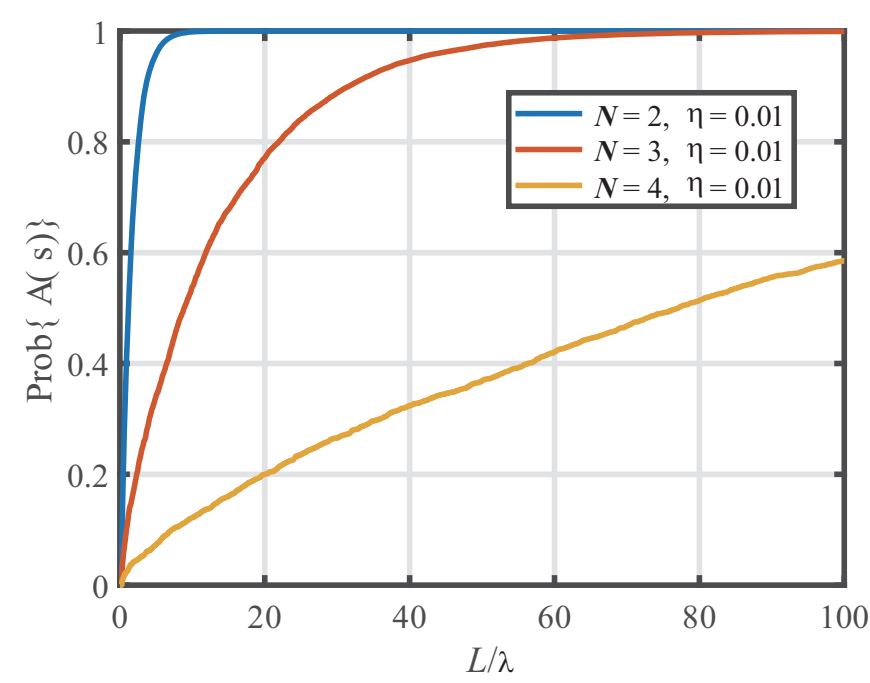

Fig. 4. $\operatorname{Prob}(A(\gamma)=1)$ of AAA-WS as a function of $L / \lambda \cdot \gamma_{\mathrm{SNR}_{\mathrm{in}}}=20$ $\mathrm{dB}$ and $\gamma_{\mathrm{SIR}_{\mathrm{in}}}=0 \mathrm{~dB}$.

where $\eta$ indicates a decrease ratio of the total interference signal power and $0<\eta<1$. We evaluate the interference suppression ability with probability of $A(\eta)=1$ while direction of arrivals $\theta_{n}$ follow i.i.d uniform distribution. The event $A(\eta)=1$ indicates that AAA-WS can suppress the interference signals sufficiently. The MRC criterion is employed for antenna spacing and weight settings.

Fig. 4 shows the probability of $A(\eta)=1$ as a function of $L$ when $\gamma_{\mathrm{SNR}_{\text {in }}}=20 \mathrm{~dB}, \gamma_{\mathrm{SIR}_{\text {in }}}=0 \mathrm{~dB}$ and $\eta=0.01$. As confirmed in Sect. V-A, the interference suppression ability of AAA-WS is enhanced by increasing $L$. Since $\eta=0.01$, the achieved attenuation level of the total interference is $20 \mathrm{~dB}$. In case of $N=2$ and $N=3, L=10 \lambda$ and $L=60 \lambda$ are necessary to achieve $\operatorname{Pro}(A(\eta)=1) \simeq 1$. In case of $N=4$, $L$ achieving $\operatorname{Pro}(A(\eta)=1) \simeq 1$ may be significantly larger than $L=100 \lambda$.

\section{B. Analysis of Outage probability}

In this section, MRC criterion is employed and possible antenna spacing is limited by $\mathbf{d}$ according to Sect. IV. An outage probability denoted by $\mathrm{P}_{\text {out }}$ is defined as follows

$$
\mathrm{P}_{\text {out }}=\operatorname{Prob}\left(P_{\min }(L)>P_{\text {thre }}\right) \text {, }
$$

where $P_{\text {thre }}$ is the target minimum interference level.

Fig. 5 shows the outage probability as a function of $K$ which is equivalent to $L$, i.e. $L=0.4 K$. The parameters in this simulation are $\gamma_{\mathrm{SNR}_{\mathrm{in}}}=20 \mathrm{~dB}$ and $\gamma_{\mathrm{SIR}_{\mathrm{in}}}=0$. In addition, $P_{\text {thre }}$ is set to $9 P_{z}$. In the MRC, achieving $P_{\min }(L)=9 P_{z}$ leads to $\gamma_{\mathrm{MRC}}(d)=10 \mathrm{~dB}$.

The result in Fig. 5 shows that larger $K$ can inherently enhance the outage probability performance in AAA-WS. The analysis can coincide with the Monte Carlo simulation results when $K$ is relatively small. The analytical result provides the lower bound of outage probability performance. The gap between the analysis and the Monte Carlo simulation results is

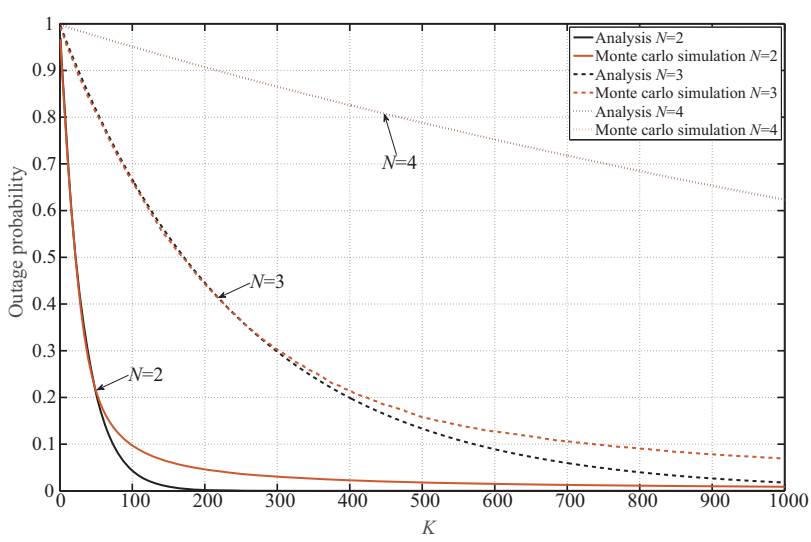

Fig. 5. Outage probability as a function of $K, \gamma_{\mathrm{SNR}_{\mathrm{in}}}=20 \mathrm{~dB}, \gamma_{\mathrm{SIR}_{\mathrm{in}}}=0$ $\mathrm{dB}, N=2, N=3$ and $N=4$, Target SINR $\gamma_{\text {thre }}=10$.

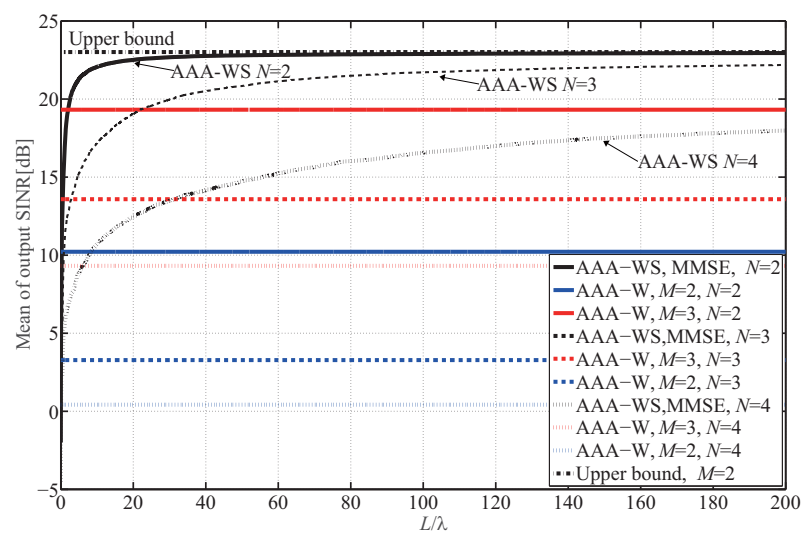

Fig. 6. Mean of SINR as a function of $L / \lambda, \gamma_{\mathrm{SNR}_{\mathrm{in}}}=20 \mathrm{~dB}, \gamma_{\mathrm{SIR}_{\mathrm{in}}}=0$ $\mathrm{dB}, N=3$. The MMSE criterion is used for the weight and antenna spacing setting.

caused by several assumptions in the analysis, such as $\delta_{n}(d)$ follows i.i.d. uniform distribution and $p_{\text {out }, n_{1}}$ and $p_{\text {out }, n_{2}}$ are independent if $n_{1} \neq n_{2}$.

\section{Comparison of interference suppression ability with the MMSE criterion}

In this section, we evaluate average SINR in terms of AAA$\mathrm{W}$ and AAA-WS with the MMSE criterion. Fig. 6 shows the average SINR as a function of $L$ for AAA-W and AAA-WS while $N=2, N=3$, and $N=4$. Upper bound SINR for $M=2$ is also shown in this figure. In the upper bound, the interference signals are assumed to be canceled perfectly and the desired signal is combined in a coherent manner. Therefore, the SINR can be $M \times \gamma_{\mathrm{SNR}_{\text {in }}}$ and it is $23 \mathrm{~dB}$ in Fig. 6.

AAA-WS with $N=2$ and $N=3$ can approximately achieve the upper bound. The gains in SINR compared to AAA-W with $N=3$ and $N=2$ are about $8 \mathrm{~dB}$ and 18 $\mathrm{dB}$, respectively, when $L / \lambda$ is more than 100 . In addition, 
additional achievable gain of AAA-WS in the region where $L / \lambda$ is more than 100 is not significant.

\section{CONCLUSION}

In this paper, we have proposed the new type of AAA, i.e. AAA with weight and antenna space control (AAA-WS). The antenna space control can improve the interference suppression ability significantly compared to AAA with weight control (AAA-W). We have shown that the interference suppression ability of AAA-WS is determined by the maximum antenna spacing $L$. In addition, we analyze the performance of AAAWS while the MRC criterion is employed and finite number of antenna spaces are assumed. The assumption in terms of finite number of antenna spaces indicates that the AAA-WS can be realized by antenna selection. Monte Carlo simulation results have implied that the analysis can provide lower bound of outage probability performance in th AAA-WS. A comparison between AAA-WS and AAA-W with MMSE criterion has shown that AAA-WS can provide significant gain in SINR, such as $18 \mathrm{~dB}$, compared to AAA-W.

\section{APPENDIX A \\ DISTRIBUTION OF $p_{\text {out }, n}$}

In this section, we derive $p_{\text {out }, n}(d)$ 's PDF $f_{n}\left(p_{\text {out }, n}\right)$ with variable antenna spacing $d$. About (21), when $\sin \theta_{n}-\sin \theta_{0}$ is an irrational number, $2 \pi \frac{d_{m_{n}}-d}{\lambda}\left(\sin \theta_{n}-\sin \theta_{0}\right) \bmod 2 \pi$ has equidistribution in $\left(\begin{array}{ll}0 & 0.5]\end{array}\right.$ [15]. So, we can re-write $p_{\text {out }, n}$ as

$$
p_{\text {out }, n}=p_{n}\{1-\cos (\Theta)\} .
$$

Here, $\Theta$ denote random variable which follows an equidistribution in $(00.5]$. The probability while $\Theta$ vary infinitesimally $1 /(2 \pi) d \Theta$ is $1 /(2 \pi) d \Theta$. So the probability while random variable vary from $p_{\text {out }, n}$ to $p_{\text {out }, n}+d p_{\text {out }, n}$ is given by (35) with consideration of that cos is even function.

$$
f_{n}\left(p_{\text {out }, n}\right)\left|d p_{\text {out }, n}\right|=2 \frac{1}{2 \pi}|d \Theta|
$$

So we can get $\left|d p_{\text {out }, n}\right|$ by calculation of diffrential (35) as follows

$$
\begin{aligned}
\left|d p_{\text {out }, n}\right| & =\left|-p_{n} \sin \Theta d \Theta\right|=p_{n} \sqrt{1-\cos ^{2} \Theta}|d \Theta| \\
& =\sqrt{p_{n}^{2}-\left(p_{\text {out }, n}-p_{n}\right)^{2}}|d \Theta| .
\end{aligned}
$$

Finally, by deformation of formula ,we can get

$$
f_{n}\left(p_{\text {out }, n}\right)=\frac{1}{\pi}\left|\frac{d \Theta}{d p_{\text {out }, n}}\right|=\frac{1}{\pi \sqrt{p_{n}^{2}-\left(p_{\text {out }, n}-p_{n}\right)^{2}}} .
$$

\section{ACKNOWLEDGMENT}

This work was supported by the European Commission in the framework of the H2020-EUJ-02-2018 project 5GEnhance (Grant agreement no. 815056) and the Ministry of Internal Affairs and Communications (MIC) of Japan.

\section{REFERENCES}

[1] A. Ghosh, T. A. Thomas, M. C. Cudak, R. Ratasuk, P. Moorut, F. W. Vook, T. S. Rappaport, G. R. MacCartney, S. Sun, and S. Nie, "Millimeter-wave enhanced local area systems: A high-data-rate approach for future wireless networks," IEEE Journal on Selected Areas in Communications, vol. 32, no. 6, pp. 1152-1163, Jun. 2014.

[2] F. Boccardi, R. W. Heath, A. Lozano, T. L. Marzetta, and P. Popovski, "Five disruptive technology directions for 5g," IEEE Communications Magazine, vol. 52, no. 2, pp. 74-80, Feb. 2014.

[3] B. Widrow, P. E. Mantey, L. J. Griffiths, and B. B. Goode, "Adaptive antenna systems," Proceedings of the IEEE, vol. 55, no. 12, pp. $2143-$ 2159 , Dec. 1967

[4] L. C. Godara, "Applications of antenna arrays to mobile communications. i. performance improvement, feasibility, and system considerations," Proceedings of the IEEE, vol. 85, no. 7, pp. 1031-1060, Jul. 1997.

[5] H. Singh and R. M. Jha, "Trends in adaptive array processing," International Journal of Antennas and Propagation, vol. 2012, 2012.

[6] J. LaMance and D. Small, "Locata correlator-based beam forming antenna technology for precise indoor positioning and attitude," Proceedings of the 24th International Technical Meeting of The Satellite Division of the Institute of Navigation (ION GNSS 2011), pp. 2436 2445, Sep. 2011.

[7] A. Moffet, "Minimum-redundancy linear arrays," IEEE Transactions on Antennas and Propagation, vol. 16, no. 2, pp. 172-175, Mar. 1968.

[8] M. B. Jorgenson, M. Fattouche, and S. T. Nichols, "Applications of minimum redundancy arrays in adaptive beamforming," IEE Proceedings $H$ - Microwaves, Antennas and Propagation, vol. 138, no. 5, pp. 441-447, Oct. 1991.

[9] L. L. Presti, G. Montalbano, and G. Povero, "Beamforming design with partially adaptive and minimum redundancy arrays," in 1996 IEEE International Conference on Acoustics, Speech, and Signal Processing Conference Proceedings, vol. 6, May 1996, pp. 3177-3180 vol. 6.

[10] L. L. Presti and G. Montalbano, "Beamforming design with adaptive variable redundancy arrays," in Military Communications Conference, 1996. MILCOM '96, Conference Proceedings, IEEE, vol. 3, Oct. 1996, pp. 863-867 vol.3.

[11] H. Duan, B. P. Ng, C. M. S. See, and J. Fang, "Broadband interference suppression performance of minimum redundancy arrays," IEEE Transactions on Signal Processing, vol. 56, no. 6, pp. 2406-2416, Jun. 2008.

[12] J. H. Winters, "Optimum combining in digital mobile radio with cochannel interference," IEEE Transactions on Vehicular Technology, vol. 33, no. 3, pp. 144-155, Aug. 1984.

[13] X. Wang, E. Aboutanios, M. Trinkle, and M. G. Amin, "Reconfigurable adaptive array beamforming by antenna selection," IEEE Transactions on Signal Processing, vol. 62, no. 9, pp. 2385-2396, May 2014.

[14] X. Wang, M. Amin, and X. Cao, "Analysis and design of optimum sparse array configurations for adaptive beamforming," IEEE Transactions on Signal Processing, vol. 66, no. 2, pp. 340-351, Jan 2018.

[15] A. Kar, "Weyl's equidistribution theorem," Resonance, Journal of Indian Academy of Sciences, 2003.

[16] C. M. Grinstead and J. L. Snell, "Chapter 7 sums of independent random variables," in Introduction to Probability: Second Revised Edition, second edition ed. American Mathematical Society, 1997, pp. 285 -304 .

[17] H. A. David and H. N. Nagaraja, Order Statistics, Third Edition, third edition ed. Wiley-Interscience, Aug. 2003. 